\title{
Convergence Results For Invariant Curve Algorithms
}

\author{
By M. van Veldhuizen
}

\begin{abstract}
In this paper a convergence result for the algorithm described by Kevrekidis et al. [7] is given. It is shown that this algorithm for the approximation of an invariant curve converges provided the curve is attracting. The approximation error is estimated. Numerical examples for three different algorithms in this class and a closely related one illustrate the theory.
\end{abstract}

Introduction. In this paper we consider a map $\Phi$ from $\mathbb{R}^{d}$ and $\mathbb{R}^{d}$ with the property that $\Phi \gamma \subset \gamma$ for a closed curve $\gamma$. This invariant curve $\gamma$ is approximated using an algorithm essentially due to Kevrekidis et al. [7]. The convergence of this algorithm under suitable conditions is the main topic of this paper.

Invariant curves and invariant manifolds arise in the study of oscillatory motion in ordinary differential equations. A typical example is given by

$$
\frac{d x}{d t}=g(x)+f(t)
$$

where $x(t) \in \mathbb{R}^{d}$, and where $f$ is a smooth periodic map with period $p$. Under reasonable conditions the differential equation (1.1) possesses a unique solution $x\left(t ; x_{0}\right)$ on $[0, p]$ with prescribed vector $x_{0}$ at $t=0$. The Poincaré map $\mathbf{P}$ with time step $p$ is defined by

$$
\mathbf{P}: y \rightarrow x(p ; y)
$$

Thus, the map $\mathbf{P}$ maps (part of) $\mathbb{R}^{d}$ to $\mathbb{R}^{d}$. The Poincaré map describes how $\mathbb{R}^{d}$ is transformed by the differential equation (1.1) in one period $p$ of the periodic forcing term $f$. A fixed point of the Poincare map $\mathbf{P}$ corresponds to a periodic solution. In some instances the oscillatory behavior of solutions of (1.1) is not periodic, but quasi-periodic. This may happen if the Poincaré map possesses an invariant closed curve $\gamma$. If $\gamma$ is a closed smooth invariant curve of $\mathbf{P}$, then the restriction $\left.\mathbf{P}\right|_{\gamma}$ of the map $\mathbf{P}$ to $\gamma$ makes sense. Observe that the set of all points

$$
\{x(t ; y) \mid t \in[0, p], y \in \gamma\}
$$

is a torus. The restriction $\left.\mathbf{P}\right|_{\gamma}$ corresponds to the restriction of the differential equation (1.1) to this torus. Since differential equations on the torus are well understood, this results in a rather complete description of the map $\left.\mathbf{P}\right|_{\gamma}$. In particular, under reasonable assumptions, $\left.\mathbf{P}\right|_{\gamma}$ is a homeomorphism from the curve $\gamma$ onto itself. Since $\gamma$ is topologically a circle, one calls $\left.\mathbf{P}\right|_{\gamma}$ the circle map. Such

Received September 11, 1986; revised September 25, 1987.

1980 Mathematics Subject ('lassification (1985 Revision). Primary 65L99; Secondary 34C05.

Key words and phrases. Invariant curve. 
homeomorphic maps from the circle onto itself are characterized by the rotation number. Roughly speaking, the rotation number of a circle map describes a kind of average rotation angle characteristic for the circle map. A rational rotation number corresponds to a fixed point of some integer power of $\left.\mathbf{P}\right|_{\gamma}$, i.e., a periodic solution of (1.1). An irrational rotation number corresponds to quasi-periodic solutions of (1.1). One should realize that the rotation number depends continuously on parameters in the Poincaré map, but not necessarily in a differentiable way. A nice mathematical description of these results and many related results may be found in Guckenheimer and Holmes [5]. The paper by Levinson [9] is still a good introduction to the existence theory for invariant curves. Hale [6] gives more general results extending many of the techniques found in Levinson's paper.

Before going on, it may be good to realize what an invariant curve may be in terms of a differential equation like (1.1). A good example is the periodically forced Van der Pol equation; asymptotic results are described in Guckenheimer and Holmes [5], and sketches of the invariant curves are given in Thoulouze-Pratt [13] and [15]. For the Van der Pol equation the invariant curve is attracting with a source (repellent periodic solution) in the interior. For many parameter values the curve is smooth and convex. For a region in parameter space the curve contains a sink and a saddle. That is, the invariant curve is a heteroclinic orbit. Let $y_{\text {saddle }}, y_{\text {sink }}$ denote the saddle and the sink, respectively. For the heteroclinic orbit each curve segment between the saddle and the sink corresponds to a solution of the recurrence equation $y_{i+1}=\mathbf{P} y_{i}$ on the set of all integers with boundary conditions at $\pm \infty$ given by $y_{\text {saddle }}$ and $y_{\text {sink }}$, respectively. Thus, an invariant curve may contain fixed points and orbits of differential equations on the real line.

Clearly, invariant curves and the restriction of the differential equation to the invariant curve play a part in the analysis of ordinary differential equations. In our terminology, the map $\Phi$ is just the Poincaré map $\mathbf{P}$. However, the restriction in the choice of $\Phi$ as a Poincaré map is not necessary. In fact, many recurrence relations give rise to invariant curves, cf. the examples in Koçak [8]. Aronson et al. [1] investigate the delayed logistic map in some detail. We use this map in Section 3.

In many instances an invariant curve cannot be obtained by analytic means. In some instances a good approximation may be obtained by averaging techniques, but even then numerical computations are often indispensable. An example of an invariant curve and a mixed asymptotic-numerical approximation procedure is given in Bouc, Defilippi and Iooss [2]. Numerical processes are already described in Thoulouze-Pratt and Jean [14], Thoulouze-Pratt [13]. However, the process mentioned in [14] may fail for problems with an almost rational rotation number. The process mentioned in [13] approximates the parametrized invariant curve by a truncated Fourier series by minimizing the distance between the original and the image under $\Phi$. This is very much like the process alluded to at the end of [15]. This process may converge to a spurious solution, even for an almost correct initial curve. The processes described by Chan [3] are based on a collocation approximation of the invariant curve. In one process the curve is parametrized such that the circle map becomes a rigid rotation. The other process relaxes this a bit. No convergence 
proofs are available, and the numerical results for these methods are not encouraging. The method described by Kevrekidis et al. [7] approximates the invariant curve by a polygon-like curve. Finally, in [15] an invariant curve is approximated by a polygon without some of the restrictions required in the Kevrekidis algorithm. In [15] there is also some discussion of algorithmic and programming details. In view of the fact that some algorithms may converge to wrong solutions, it is desirable to have algorithms with proven convergence under suitable conditions. The Kevrekidis algorithms (described in Section 2) seem a good candidate for investigating the convergence properties. Their formulation and implementation does not depend on the rotation number of the circle map corresponding to the invariant curve to be approximated. So the rationality or irrationality of the rotation number does not interfere with the approximating process. For attractive invariant curves we may use a contraction argument. In this way we mimic the theory of Levinson [9] for a discretized problem. An approach via the Newton method is not feasible: the corresponding map is only piecewise differentiable. The approach presented here has a disadvantage. We can only deal with attractive invariant curves or repellent ones. For curves of mixed stability type the analysis fails. A dichotomy argument as in Hale [6] seems necessary, but it is far from clear how to do it.

In this paper we analyze the algorithm of Kevrekidis et al. [7]. We are able to show that the simplest method converges under fairly general conditions. Higherorder methods require special assumptions. This theory is given in Section 2. In Section 3 we give some examples. Four methods are described in some detail. A simple test problem is analyzed and used in the numerical experiments. And the four methods are applied to the delayed-logistic map, cf. Aronson et al. [1].

Details on the implementation are given in [15] for a slightly different method. The numerical approximation of the rotation number is investigated to some extent in [16].

2. Convergence Analysis. In this section we describe algorithms for the numerical approximation of an invariant curve of a map $\Phi$. In all instances we assume that the restriction of $\Phi$ to $\gamma$ is a homeomorphism. First, we describe two nonlinear coordinate transformations, and we give a simple property. One of these coordinate transformations is the basis for the algorithms. The main result of this section is the convergence analysis of the algorithms for an attractive invariant curve. For the algorithm based on piecewise linear interpolation we obtain a satisfactory convergence result. For higher-order methods we obtain convergence results under some restrictions. The results of the discussion are summarized in Theorems 2.6, 2.7, and 2.9 below.

Let the simple closed curve $\gamma \in \mathbb{R}^{d}$ be an invariant curve of the map $\Phi$. In $\mathbb{R}^{2}$ the curve $\gamma$ is a Jordan curve. The approximation of the curve $\gamma$ will be based on a polygon in $\mathbb{R}^{d}$. A polygon is completely determined by its vertices. The $N$ vertices, vectors in $\mathbb{R}^{d}$, are denoted by $x_{1}, x_{2}, \ldots, x_{N}$, and the polygon $\mathbf{p}\left(\left\{x_{i}\right\}_{i=1}^{N}\right)$ is obtained as the set of line segments $\left[x_{1}, x_{2}\right],\left[x_{2}, x_{3}\right], \ldots,\left[x_{N-1}, x_{N}\right]$ and $\left[x_{N}, x_{1}\right]$. For the analysis we need a different coordinate system. Since it is so fundamental, we introduce this nonlinear coordinate transformation first. 
Assumption 2.1 (tubular coordinates). Any vector $x$ in an annular neighborhood of $\gamma \in \mathbb{R}^{d}$ can be written as

$$
x=u(\tau)+Z(\tau) \xi
$$

where $\tau \in[0,2 \pi), \xi \in \mathbb{R}^{d-1}$, and $Z(\tau)$ is a $d \times(d-1)$ matrix with orthonormal column vectors. In addition, all columns of $Z(\tau)$ are orthogonal to the vector $\frac{d u}{d \tau}(\tau)$. We may even assume that the length of the vector $\frac{d u}{d \tau}$ is independent of $\tau$.

Let us consider this assumption in some detail. Clearly, (2.1) defines a nonlinear coordinate transformation $x \rightarrow(\tau, \xi)$. In the new coordinates the invariant curve is given by $\tau \rightarrow(\tau, 0)$, corresponding to the parametrization $\tau \rightarrow u(\tau)$ in the original coordinates. The orthogonality of the augmented matrix $Z$ implies that the vector $\xi$ measures the deviation from the curve in a hyperplane orthogonal to the tangent vector to $\gamma$ in $u(\tau)$. In $\mathbb{R}^{2}$ the matrix $Z$ reduces to the normal vector to the curve. In the sequel we mean by the distance $d(x ; \gamma)$ of a vector $x$ to $\gamma$ the Euclidean norm $\|\xi\|$.

The nonlinear coordinate transformation is not always well defined. However, for important classes of problems, Hale [6] proves that the transformation is well defined, i.e., in an annular neighborhood of the simple closed curve $\gamma$ the transformation is a smooth invertible map. See also Levinson [9], who may have been the first to use a coordinate system like (2.1). For instance, the transformation (2.1) is well defined for problems like (1.1) in a neighborhood of $f=0$, provided the autonomous system $x^{\prime}=g(x)$ has a stable periodic solution.

In $\mathbb{R}^{2}$ the nonlinear transformation (2.1) requires only a point in the interior of the Jordan curve $\gamma$. In $\mathbb{R}^{d}, d>2$, one needs some knowledge about the position of the one-dimensional manifold $\gamma$ in $d$-space. As rigorously proved by Hale [6], for a simple closed curve $\gamma$ there always exists a vector not in the span of the set of all tangent vectors to $\gamma$ (the idea goes back to Levinson [9]). For example, if the curve $\gamma$ in $\mathbb{R}^{3}$ is in the $x, y$-plane, then the $z$-axis is not in the span of all tangent vectors to $\gamma$. Hale's result says that this situation is typical. In practice one might use asymptotic information for finding a good candidate for the vector not in the span of all tangent vectors. If $\gamma$ is parametrized by $\tau \rightarrow u(\tau)$, we assign to a vector $x$ in a neighborhood of $\gamma$ the pair $(\tau, x-u(\tau))=(\tau, Z(\tau) \xi)$, with $\tau$ chosen such that $x-u(\tau)$ is orthogonal to the tangent vector to $\gamma$ in $\tau$. The mathematical details of this construction are given by Hale [6]. By the attractivity of the invariant curve $\gamma$ we mean the existence of a constant $0 \leq \kappa<1$ such that for all $x$ in an annular neighborhood of $\gamma$

$$
d(\Phi x ; \gamma) \leq \kappa d(x ; \gamma)
$$

The attractivity of the curve $\gamma$ is expressed by means of the tubular coordinates introduced above. Tubular coordinates are essential in describing an important property of the curve $\gamma$. But for the algorithm we need another coordinate system. We assume that the invariant curve $\gamma$ looks very much like a curve in a plane $\mathbf{R}$. Since we need this plane in the algorithm, we may as well assume that $\mathbf{R}$ is spanned by the first two coordinate axes in $\mathbb{R}^{d}$. We can always achieve this by a linear transformation. Henceforth we assume for some $x_{C} \in \mathbf{R}$ the existence of the following nonlinear coordinate transformation. 
Assumption 2.2 (radial coordinates). In an annular neighborhood of the curve $\gamma$ the nonlinear coordinate transformation

$$
(\theta, \varrho, \eta) \rightarrow x_{C}+(r(\theta)+\varrho)(\cos (\theta), \sin (\theta), 0)^{T}+(0,0, \varsigma(\theta)+\eta)^{T}
$$

is a smooth invertible map, and $r(\theta)>0$. In particular, the Jacobian matrix of the transforming map should be invertible with uniformly bounded inverse along $\gamma$.

Under this assumption the curve $\gamma$ is described by

$$
\gamma: \theta \in[0,2 \pi) \rightarrow \gamma(\theta)=x_{C}+(r(\theta) \cos (\theta), r(\theta) \sin (\theta), \varsigma(\theta))^{T} .
$$

If $d=2$ the $\zeta$-coordinate in (2.3), (2.4) is absent, and the formulation simplifies. So the Euclidean projection of $\gamma$ onto $\mathbf{R}$ is a star-shaped curve with respect to $x_{C}$ in its interior. Not all smooth simple curves in $\mathbb{R}^{d}$, or even $\mathbb{R}^{2}$, may be represented in this way. The restriction is motivated by the algorithm to be presented below. These coordinates are very much like polar coordinates centered at $x_{C}$.

Clearly, Assumption 2.2 implies Assumption 2.1, showing that we are somewhat restrictive. In practice this is not too serious a limitation. Many invariant curves arise from a Hopf bifurcation. The curve in $\mathbb{R}^{4}$ approximated by Bouc et al. [2] originates in a Hopf bifurcation. In such a case the essential action takes place in the plane determined by the eigenvectors (of the Jacobian matrix of $\Phi$ ) corresponding to the two eigenvalues crossing the imaginary axis. This gives detailed asymptotic information about the plane $\mathbf{R}$.

In radial coordinates the distance $d_{\mathrm{rad}}(x ; \gamma)$ from $x=(\theta, \varrho, \eta)$ to $\gamma$ is given by the Euclidean length of the vector $(\varrho, \eta)$. We compare this distance with the distance in tubular coordinates.

LEMMA 2.3. There exists a constant $C_{d} \geq 1$ such that for all $x$ in an annular neighborhood of $\gamma$, we have

$$
d(x ; \gamma) \leq d_{\mathrm{rad}}(x ; \gamma) \leq C_{d} d(x ; \gamma) .
$$

The constant $C_{d}$ depends on the position of $x_{C}$ in the interior of the projection of $\gamma$ onto $\mathbf{R}$ and the size of the annular neighborhood.

Proof. The first inequality is obvious. The second inequality follows from the uniform boundedness of the inverse Jacobian matrix of the coordinate transformation along $\gamma$. See also Figure 3.2.

Remark 2.4. Observe that the value of $C_{d}$ depends only on the properties of $\gamma$ and the radial coordinates. For example, if we use $\Phi^{p}$ instead of $\Phi$ we find the same value for $C_{d}$.

We now return to the polygon. The images under $\Phi$ of the vertices $x_{i}$ form a polygon $\mathbf{p}\left(\left\{\Phi x_{i}\right\}_{i=1}^{N}\right)$. For an attracting curve $\gamma$ we expect that $\mathbf{p}\left(\left\{\Phi x_{i}\right\}_{i=1}^{N}\right)$ is a better approximation than $\mathbf{p}\left(\left\{x_{i}\right\}_{i=1}^{N}\right)$. As in [15] we project the old polygon $\mathbf{p}\left(\left\{x_{i}\right\}_{i=1}^{N}\right)$ on the new polygon $\mathbf{p}\left(\left\{\Phi x_{i}\right\}_{i=1}^{N}\right)$ by means of a projection. Here we use interpolation.

The combination of $\Phi$ (considered as a map acting on the vertices of $\left.\mathbf{p}\left(\left\{x_{i}\right\}_{i=1}^{N}\right)\right)$ and the projection will be denoted by $K$. We describe $K$ in the case $d=2$. Let $x_{i}$ be a vertex of $\mathbf{p}\left(\left\{x_{i}\right\}_{i=1}^{N}\right)$. Determine the angle $\theta_{i}$ such that

$$
x_{i}=x_{C}+\left\|x_{i}-x_{C}\right\|\left(\cos \left(\theta_{i}\right), \sin \left(\theta_{i}\right)\right)^{T} .
$$


Assume that the point $x_{C}$ is also in the interior of the polygon $\mathbf{p}\left(\left\{\Phi x_{i}\right\}_{i=1}^{N}\right)$. This is so if the polygon $\mathbf{p}\left(\left\{x_{i}\right\}_{i=1}^{N}\right)$ is close enough to $\gamma$. The vertices of the polygon $\mathbf{p}\left(\left\{\Phi x_{i}\right\}_{i=1}^{N}\right)$ may then be written in this coordinate system as

$$
\Phi x_{j}=x_{C}+\hat{r}_{j}\left(\cos \left(\hat{\theta}_{j}\right), \sin \left(\hat{\theta}_{j}\right)\right)^{T} .
$$

Assume $\theta_{i} \in\left[\hat{\theta}_{j}, \hat{\theta}_{j+1}\right]$, taking into account the identification of $2 \pi$ and 0 . Define the $i$ th vertex of $K=K_{l}$ as the intersection of the half-line in the direction $\theta_{i}$ and the line segment $\left[\Phi x_{j}, \Phi x_{j+1}\right]$,

$$
K_{l} x_{i}=\left\{x \mid x=x_{C}+r\left(\cos \left(\theta_{i}\right), \sin \left(\theta_{i}\right)\right)^{T}, r>0\right\} \cap\left[\Phi x_{j}, \Phi x_{j+1}\right] .
$$

See also Figure 2.1. In $\mathbb{R}^{d}, d>2$, we intersect with the hyperplane determined by the direction $\theta_{i}$ in $\mathbf{R}$, i.e., a half-line through $x_{C}$, and the orthogonal complement of $\mathbf{R}$. In this method the approximation to $\gamma$ is a polygon.

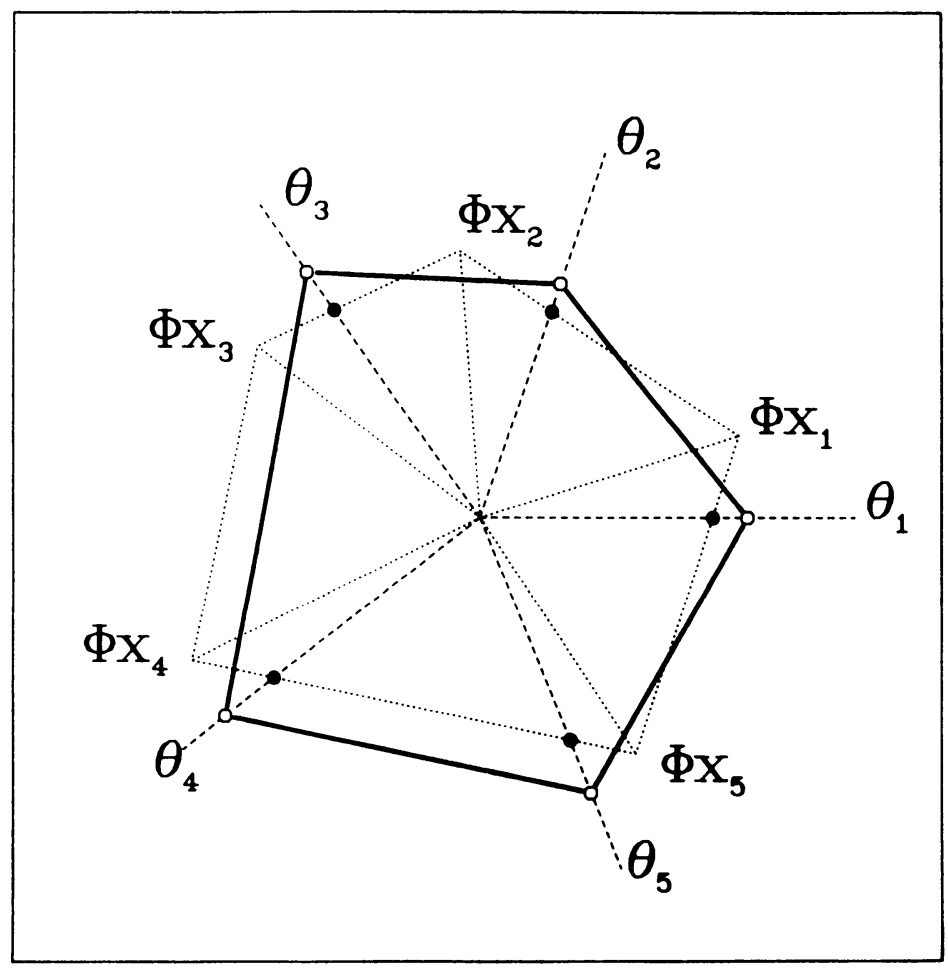

FIGURE 2.1

Construction of $K_{l}$ for $N=5$. The spokes are indicated by the dashed lines and the angles $\theta_{i}$. The polygon with its vertices on the spokes is indicated by the heavy lines. The vertex (open dot) $x_{i}$ lies on the spoke indicated by $\theta_{i}$. The polygon of the images is shown in dotted lines. So are the half-lines determined by the images $\Phi x_{i}$. The vertices of the new polygon, the points $K_{l} x_{i}$, are indicated by the heavy dots. The new polygon is not shown.

Different maps $K$ are possible and useful. We might interpolate in radial coordinates with abscissae on the $\theta$-axis. Then define the $i$ th vertex $K x_{i}$ of the polygon $K \mathbf{p}\left(\left\{x_{i}\right\}_{i=1}^{N}\right)$ by interpolation by the operator $\Pi$ in the point $\theta_{i}$. Thus we interpolate with values in the abscissae $\hat{\theta}_{j}$. We consider $\Pi$ as a projection operator in the Banach space of continuous functions on $[0,2 \pi)$ equipped with the usual supremum 
norm of functions. The norm in the Banach space is denoted by || . The norm of $\Pi$ in the Banach space of continuous functions on $[0,2 \pi)$ is denoted by $|\Pi| ;|\Pi| \geq 1$ may depend strongly on the distribution of the abscissae $\hat{\theta}_{j}$ and $N$. By $\Pi \gamma$ we mean interpolation of the curve $\gamma$ itself in the abscissae $\hat{\theta}_{j}$. We assume $\Pi \gamma \rightarrow \gamma$ for $N \rightarrow \infty$. The approximating polygon is defined as the solution $\mathbf{p}\left(\left\{\bar{x}_{i}\right\}_{i=1}^{N}\right)$ (if any) of the set of equations $\mathbf{p}\left(\left\{x_{i}\right\}_{i=1}^{N}\right)=\mathbf{p}\left(\left\{K x_{i}\right\}_{i=1}^{N}\right)$. The approximation to $\gamma$ is then given by $\Pi \mathbf{p}\left(\left\{\bar{x}_{i}\right\}_{i=1}^{N}\right)$. All this carries over directly to the case $d>2$. With piecewise linear interpolation and $d=2$ we recover the scheme by Kevrekidis et al. [7]. See also Figure 2.2.

The map $K_{l}$ may be viewed as composition of $\Phi$ and some kind of nonlinear interpolation. So the above framework seems not applicable, but see Remark 2.10 .

In order to simplify the discussion and the notation, we shall continue in $\mathbb{R}^{2}$. At appropriate places the modifications required for $\mathbb{R}^{d}, d>2$, will be pointed out.

The interpolation is well defined for polygons sufficiently close to $\gamma$ with $x_{C}$ in the interior of $\gamma$ (or its projection on $\mathbf{R}$ ). Consider a polygon such that the $\theta_{i}$ form a strictly monotone sequence (after lifting them to the real line). Since the restriction of $\Phi$ to $\gamma$ is a homeomorphism by assumption, the images $\Phi u\left(\theta_{i}\right)$ are again nicely ordered along $\gamma$. So, if the vertices are close enough to $\gamma$, then the images of the vertices are also in order along $\gamma$. Thus the position of $x_{i}$ is uniquely determined in relation to the $\Phi x_{j}$.

Observe that the interpolation process may fail to interpolate in some subintervals, while doing more than one interpolation in another one. It all depends on the distribution of the $\hat{\theta}_{j}$ in relation to the $\theta_{i}$. Hence, some vertices may play a more prominent role in the process than others.

Kevrekidis et al. [7] suggest Newton iteration for solving the equations. This might work in many instances, but the map $K$ is not necessarily differentiable. Consider interpolation by continuous, not necessarily differentiable piecewise polynomials. A loss of differentiability of $K$ occurs if small variations in the vertex $x_{j}$ cause $\hat{\theta}_{j}$ to cross the value $\theta_{i}$. Then, for one $x_{j}$ we must interpolate in $\left[\hat{\theta}_{j}, \hat{\theta}_{j+1}\right]$, and for slightly different value we must interpolate in $\left[\hat{\theta}_{j-1}, \hat{\theta}_{j}\right]$. Clearly, this results in a discontinuity in the derivative of $K$. Therefore we restrict ourselves to stable invariant curves, and we solve the equation $\mathbf{p}\left(\left\{x_{i}\right\}_{i=1}^{N}\right)=\mathbf{p}\left(\left\{K x_{i}\right\}_{i=1}^{N}\right)$ by iteration.

A basic step of the algorithm consists of three parts: compute the images of the vertices of the current polygonal approximation $\mathbf{p}\left(\left\{x_{i}\right\}_{i=1}^{N}\right)$; project the old vertices onto the polygon $\mathbf{p}\left(\left\{\Phi x_{i}\right\}_{i=1}^{N}\right)$ and define a new polygon by taking the projections of the old vertices as the vertices. In view of the definition of the map $K$, it is obvious that the vertices of the successive polygonal approximations are of the form

$$
x_{i}^{(j)}=x_{C}+r_{i}^{(j)}\left(\cos \left(\theta_{i}\right), \sin \left(\theta_{i}\right)\right)^{T},
$$

where the angle $\theta_{i}$ is independent of the number of iterations. That is, the angles $\theta_{i}$ are determined by the initial guess only. The half-lines determined by these angles look like spokes in a wheel. The relative position of these spokes determines to a large extent the success of the method. In a practical algorithm the relative position should be determined in an adaptive manner. In the convergence analysis we shall consider the case of $N$ fixed spokes nicely distributed in all directions. The directions given by the $\hat{\theta}_{j}$, cf. (2.6), do depend on the iteration steps. This means 
that the norm of $\Pi$ may vary with varying interpolation abscissae. In a sufficiently small annular neighborhood of $\gamma$ the variations in $\hat{\theta}_{j}$ are bounded. Henceforth we use the maximum of the norm of $\Pi$ over all such possible sets of interpolation abscissae. We denote it by $|\Pi|_{\max }$. All this holds true almost literally for the algorithm in $\mathbb{R}^{d}, d>2$.

We want to apply the Brouwer fixed-point theorem. To that end, we start with a lemma.

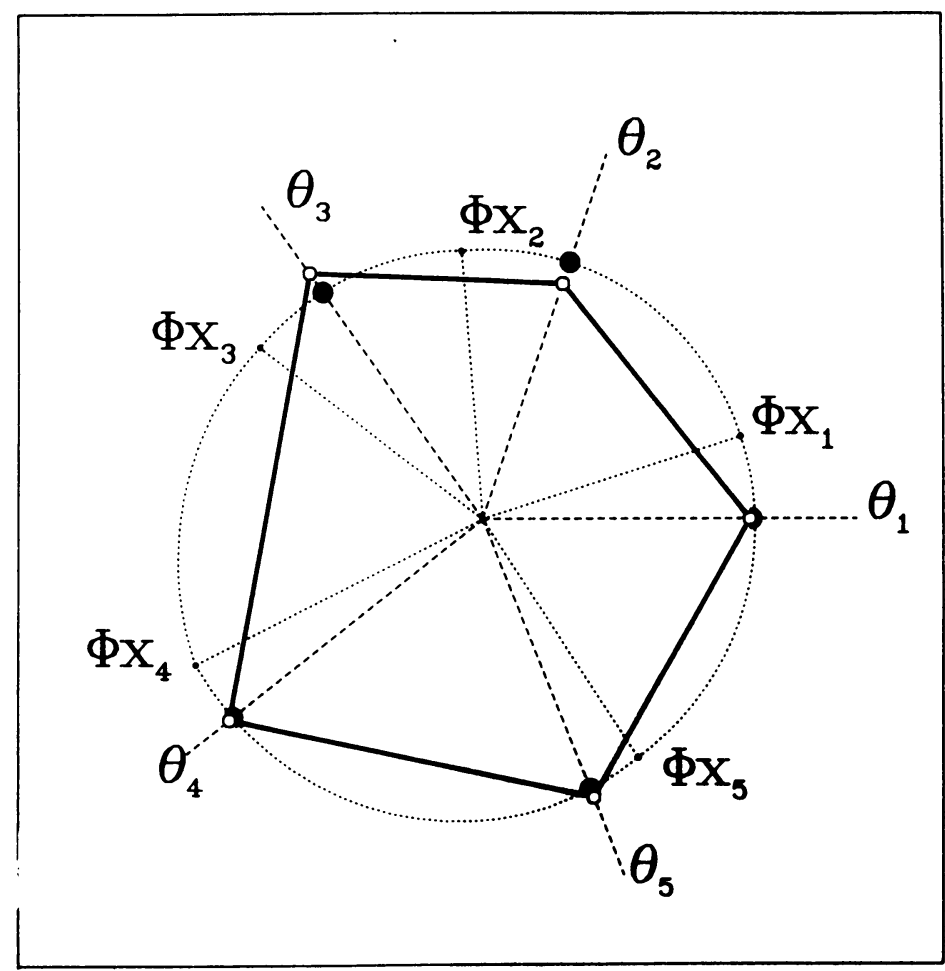

FIGURE 2.2

Construction of $K$ for $N=5$ and piecewise linear interpolation. The spokes are indicated by the dashed lines and the angles $\theta_{i}$. The polygon with its vertices on the spokes is indicated by the heavy lines. The vertex (open dot) $x_{i}$ lies on the spoke indicated by $\theta_{i}$. The curve $\Pi p\left(\left\{\Phi x_{i}\right\}_{i=1}^{N}\right)$ is indicated by a dotted line. So are the half-lines determined by the directions $\hat{\theta}_{i}$. The vertices of the new polygon, the points $K x_{i}$, are indicated by the heavy dots. The new polygon is not shown. See also Figure 2.1.

LEMMA 2.5. For $\mathbf{p}\left(\left\{x_{i}\right\}_{i=1}^{N}\right)$ close enough to $\gamma$,

$$
d_{\mathrm{rad}}\left(K x_{i} ; \gamma\right) \leq \kappa C_{d}|\Pi|_{m} \max _{i=1, \ldots, N} d\left(x_{i} ; \gamma\right)+|\Pi \gamma-\gamma| \text {. }
$$

Proof. The radial distance between $K x_{i}$ and $\gamma$ is estimated by

$$
d_{\mathrm{rad}}\left(K x_{i} ; \gamma\right) \leq\left|\Pi \mathbf{p}\left(\left\{\Phi x_{i}\right\}_{i=1}^{N}\right)-\Pi \gamma\right|+|\Pi \gamma-\gamma| \text {. }
$$

The latter term is the norm of the interpolation error. The former may be estimated as, cf. Lemma 2.3,

$$
\left|\Pi \mathbf{p}\left(\left\{\Phi x_{i}\right\}_{i=1}^{N}\right)-\Pi \gamma\right| \leq|\Pi| \max _{i} d_{\mathrm{rad}}\left(\Phi x_{i} ; \gamma\right) \leq|\Pi| C_{d} \max _{i} d\left(\Phi x_{i} ; \gamma\right) .
$$

By using the attractivity of $\gamma$ and the definition of $|\Pi|_{m}$, the result is obtained. 
We shall determine a convex set mapped by $K$ into itself. Consider a polygon $\mathbf{p}\left(\left\{x_{i}\right\}_{i=1}^{N}\right)$ and assume that the radial distance of the vertices to $\gamma$ is at most $\delta>0$. We want to show that the iterates of $\mathbf{p}\left(\left\{x_{i}\right\}_{i=1}^{N}\right)$ have the same property, provided we are close to $\gamma$ with $N$ sufficiently large. Since $K x_{i}$ and $x_{i}$ belong to the same spoke, and since the radial distance of $K x_{i}$ to $\gamma$ is estimated by Lemma 2.5, the condition

$$
\delta<\kappa C_{d}|\Pi|_{m} \delta+|\Pi \gamma-\gamma|
$$

suffices.

THEOREM 2.6. If $\kappa C_{d}|\Pi|<1$ and if the interpolation error is sufficiently small, then the equation $\mathbf{p}\left(\left\{x_{i}\right\}_{i=1}^{N}\right)=\mathbf{p}\left(\left\{K x_{i}\right\}_{i=1}^{N}\right)$ has at least one solution in the closure of an annular neighborhood of $\gamma$. The radial distance from points in this neighborhood to $\gamma$ is bounded by

$$
\delta=\frac{|\Pi \gamma-\gamma|}{1-\kappa C_{d}|\Pi|_{m}}
$$

Proof. Under the assumptions of the theorem, condition (2.9) is satisfied. Thus, the map $K$ consists of $N$ interrelated maps from line segments on spokes to line segments on spokes. These line segments may be viewed as an $N$-cube in $\mathbb{R}^{N}$. Thus $K$ maps an $N$-cube into itself. In radial coordinates, the smallest possible neighborhood is contained in the one with diameter $\delta$ as given by (2.10). In the general case, we have a $(d-1) N$-cube, but the arguments are identical. Clearly, $K$ is continuous. Hence, by the Brouwer fixed-point theorem there is at least one solution for the equations $\mathbf{p}\left(\left\{x_{i}\right\}_{i=1}^{N}\right)=\mathbf{p}\left(\left\{K x_{i}\right\}_{i=1}^{N}\right)$.

THEOREM 2.7. In addition to the assumptions of Theorem 2.6, let $\Pi$ be the piecewise linear interpolation operator (on the abscissae $\hat{\theta}_{j}$ ), and let the interpolation error be sufficiently small. Then the equation has a unique solution $\mathbf{p}\left(\left\{\bar{x}_{i}\right\}_{i=1}^{N}\right)$ and the sequence $\mathbf{p}\left(\left\{x_{i}\right\}_{i=1}^{N}\right), \mathbf{p}\left(\left\{K x_{i}\right\}_{i=1}^{N}\right), \mathbf{p}\left(\left\{K^{2} x_{i}\right\}_{i=1}^{N}\right), \ldots$ converges to it with a convergence factor $\leq \kappa^{\prime}$, where $C_{d} \kappa<\kappa^{\prime}<1$.

Proof. Locally, in a neighborhood of $\gamma$, we have by Lemma 2.3

$$
d_{\mathrm{rad}}(\Phi x ; \gamma) \leq C_{d} \kappa d_{\mathrm{rad}}(x ; \gamma)
$$

In radial coordinates this property implies that the $(\varrho, \eta)$-component of the derivative of $\Phi$ in the direction $(\varrho, \eta)$, cf. (2.3), for points on $\gamma$ is bounded by $C_{d} \kappa<1$. Partition the Jacobian matrix $J_{\Phi}(x)$ of $\Phi$ (in radial coordinates) according to $\theta$ (first part of partitioning) and $(\varrho, \eta)$ (second part),

$$
J_{\Phi}(x)=\left(\begin{array}{ll}
J_{1,1}(x) & J_{1,2}(x) \\
J_{2,1}(x) & J_{2,2}(x)
\end{array}\right) .
$$

Then also $\left\|J_{2,2}(x)\right\| \leq \kappa C_{d}$ for all $x \in \gamma$.

Let $\mathbf{p}\left(\left\{\bar{x}_{i}\right\}_{i=1}^{N}\right)$ be a solution as given by Theorem 2.6. Consider $\Phi x_{j}$ for some vertex $x_{j}$ close to the vertex $\bar{x}_{j}$. Then

$$
\Phi x_{j}-\Phi \bar{x}_{j}=\int_{0}^{1} J_{\Phi}\left(\bar{x}_{j}+s \Delta x_{j}\right) \Delta x_{j} d s
$$


with $\Delta x_{j}=x_{j}-\bar{x}_{j}$. By continuity and by the estimate for $\left\|J_{2,2}\right\|$, there is an $\varepsilon>0$ such that the radial component of $\Phi x_{j}-\Phi \bar{x}_{j}$ satisfies the estimate

$$
\| \text { radial component }\left(\Phi x_{j}-\Phi \bar{x}_{j}\right)\left\|\leq\left(\kappa C_{d}+\varepsilon\right)\right\| \Delta x_{j} \|
$$

where $\varepsilon \rightarrow 0$ for $\Delta x_{j} \rightarrow 0$ and $\bar{x}_{j} \rightarrow \gamma$. Thus, if the interpolation error is sufficiently small, there is an annular neighborhood of $\gamma$ with

$$
\| \text { radial component }\left(\Phi x_{j}-\Phi \bar{x}_{j}\right)\left\|\leq \kappa^{\prime}\right\| \Delta x_{j} \| \text {. }
$$

Since we use piecewise linear interpolation, the distance from $K x_{i}$ obtained by interpolation of $\Phi x_{j}$ and $\Phi x_{j+1}$ has a radial distance to $K \bar{x}_{i}=\bar{x}_{i}$ of at most $\kappa^{\prime} \max _{j}\left\|\Delta x_{j}\right\|$. We must take the maximum over all possible differences, because $K x_{i}$ and $K \bar{x}_{i}$ may use different abscissae in the interpolation stage. Thus,

$$
\max _{i}\left\|K x_{i}-K \bar{x}_{i}\right\| \leq \kappa^{\prime} \max _{j}\left\|\Delta x_{j}\right\| .
$$

Hence $K$ is a contraction. The assertions now follow immediately from the contraction principle.

This result says that the method with piecewise linear interpolation has a unique solution provided the curve $\gamma$ is an attractive invariant curve in the radial coordinate system. Of course, Remark 2.4 is rather relevant if $\kappa$ is not so small or if $C_{d}$ is large.

The result is based on a simple property of line segments, i.e., piecewise linear interpolation. For more complicated interpolation schemes there still is at least one solution (if $\|\Pi\|_{m}$ is not too large). But if we argue as in the proof of Theorem 2.7, we must compare two interpolation results, one for $\bar{x}_{i}$, one for $K x_{i}$, obtained on different abscissae with data of which only components in radial directions have known bounds. Without additional assumptions about the interpolation scheme or the abscissae $\hat{\theta}_{j}$, no results seem possible. As a way out, we make a simple assumption.

Assumption 2.8. In a sufficiently small annular neighborhood of $\gamma$ we have

$$
\|\Phi x-\Phi y\| \leq \bar{\kappa}\|x-y\|
$$

for $x, y$ vectors with the same $\theta$-coordinate (on the same half-line centered in $x_{C}$ ).

This assumption limits the size of the component $J_{2,1}$ in the above partitioned Jacobian matrix (2.11). Equivalently, it restricts the action of $\Phi$ parallel to $\gamma$. Clearly, $\bar{\kappa} \geq \kappa$. See Section 3 for an example in which $\bar{\kappa}, \kappa^{\prime}$ can be computed explicitly. With this restricting assumption we have the following result.

THEOREM 2.9. Under the above assumptions, in particular Assumption 2.8, let $\mathbf{p}\left(\left\{x_{i}\right\}_{i=1}^{N}\right)$ belong to a sufficiently small annular neighborhood of $\gamma$. Let $\Pi$ be the operator defined by piecewise linear interpolation. Let $\bar{\kappa}|\Pi|_{m}<1$. Then the sequence $\mathbf{p}\left(\left\{x_{i}\right\}_{i=1}^{N}\right), \mathbf{p}\left(\left\{K x_{i}\right\}_{i=1}^{N}\right), \mathbf{p}\left(\left\{K^{2} x_{i}\right\}_{i=1}^{N}\right), \ldots$ converges to a unique solution $\mathbf{p}\left(\left\{\bar{x}_{i}\right\}_{i=1}^{N}\right)$ of the equation $\mathbf{p}\left(\left\{x_{i}\right\}_{i=1}^{N}\right)=\mathbf{p}\left(\left\{K x_{i}\right\}_{i=1}^{N}\right)$. The discretization error in the vertices satisfies the estimate

$$
\max _{i=1, \ldots, N} d\left(\bar{x}_{i} ; \gamma\right) \leq \frac{|\Pi \gamma-\gamma|}{1-\bar{\kappa}|\Pi|_{m}}
$$


Proof. Consider two polygons $\mathbf{p}\left(\left\{x_{i}\right\}_{i=1}^{N}\right), \mathbf{p}\left(\left\{y_{i}\right\}_{i=1}^{N}\right)$ in a sufficiently small annular neighborhood of $\gamma$. Then

$$
\begin{aligned}
\left\|K x_{i}-K y_{i}\right\| & \leq|\Pi|\left|\mathbf{p}\left(\left\{\Phi x_{i}\right\}_{i=1}^{N}\right)-\mathbf{p}\left(\left\{\Phi y_{i}\right\}_{i=1}^{N}\right)\right| \\
& \leq|\Pi| \max _{j}\left\|\Phi x_{j}-\Phi y_{j}\right\| \leq|\Pi| \bar{\kappa} \max _{j}\left\|x_{j}-y_{j}\right\| .
\end{aligned}
$$

Observe that the $i$ th vertex of the polygon $K \mathbf{p}\left\{\gamma\left(\theta_{i}\right)\right\}_{i=1}^{N}$ is given by $(\Pi \gamma)\left(\theta_{i}\right)$. Hence, for any vertex $\bar{x}_{i}$ of a solution $\mathbf{p}\left(\left\{\bar{x}_{i}\right\}_{i=1}^{N}\right)$ we have

$$
\begin{aligned}
\left\|\bar{x}_{i}-\gamma\left(\theta_{i}\right)\right\| & \leq\left\|K \bar{x}_{i}-(\Pi \gamma)\left(\theta_{i}\right)\right\|+\left\|(\Pi \gamma)\left(\theta_{i}\right)-\gamma\left(\theta_{i}\right)\right\| \\
& \leq \bar{\kappa}|\Pi|_{m} \max _{j}\left\|\bar{x}_{j}-\gamma\left(\theta_{j}\right)\right\|+|\Pi \gamma-\gamma| .
\end{aligned}
$$

From these estimates the result is easily obtained.

Remark 2.10. With some simple modifications the theory and the results carry over to the method described by the map $K_{l}$, cf. (2.7). In this case too, we may view $K_{l}$ as the composition of $\Phi$ (acting on vertices of polygons) and a projection. In this instance the projection is nonlinear. It hardly matters. We shall consider the relevant parts of the proofs of this section. In Lemma 2.6 one should interpret the interpolation error as the distance in radial coordinates between $\gamma$ and the chord connecting $\gamma\left(\hat{\theta}_{j}\right)$ and $\gamma\left(\hat{\theta}_{j+1}\right)$. This leads directly to the estimate

$$
d_{\mathrm{rad}}\left(K x_{i} ; \gamma\right) \leq \kappa \max _{i=1, \ldots, N} d\left(x_{i} ; \gamma\right)+|\Pi \gamma-\gamma| \text {. }
$$

Similarly, the statements of Theorem 2.7 and Theorem 2.9 are easily seen to hold true. The linearity of $\Pi$ is not really used.

Remark 2.11. If the restriction of $\Phi$ to $\gamma$ has fixed points (an even number), then these points divide the curve in heteroclinic orbits between successive fixed points. Suppose we know these fixed points, and we use them as vertices in the polygon. Let such a vertex, a fixed point, correspond to the radial coordinate $\theta_{i}$. Since it is a fixed point of $\Phi$, we have $\hat{\theta}_{i}=\theta_{i}$. Since interpolation of a function in an abscissa returns the function value in that abscissa, we see that the fixed point is also a fixed vertex of the sequence of polygons. As a consequence, we see that we may restrict ourselves to each heteroclinic orbit separately, provided the interpolation process can be restricted to each of the subintervals separately. This is so for piecewise linear interpolation. Then the above theory also applies, since it does not mix up the various heteroclinic orbits. Consequently, the algorithms of this section may be useful in approximating heteroclinic orbits in ordinary differential equations without any problems (truncation of the integration interval, asymptotic matching) at $\pm \infty$.

3. Examples. In this section we describe a few special cases of the algorithms defined in Section 2. We describe some properties, and we give some examples. The notation of Section 2 is used in this section.

Method 3.1. This is the method described in Section 2, with $\Pi=\Pi_{1}$ the piecewise linear interpolation operator. That is, between two abscissae $\hat{\theta}_{j}$ and $\hat{\theta}_{j+1}$, cf. (2.6), we use interpolation by a polynomial of degree $\leq 1$. The corresponding operator satisfies $|\Pi|_{m}=1$ for all subdivisions of the interval $[0,2 \pi)$. Hence, for sufficiently many vertices, and if $\kappa C_{d}<1$, there is a unique solution close to $\gamma$, cf. Theorem 2.7. The approximation error is bounded by $(2.10)$, i.e., $O\left(\max _{j}\left|\hat{\theta}_{j}-\hat{\theta}_{j+1}\right|^{2}\right)$. 
This method has a simple property if $\gamma$ is a circle and if $x_{C}$ is in the center of the circle. The statement is a consequence of Theorem 2.7 and is valid for all interpolatory methods of Section 2.

LEMMA 3.2. Let the attracting invariant curve $\gamma$ be a circle. Let $x_{C}$ be the center of this circle. Then Method 3.1 is exact (approximation $=\gamma$ ), and the vertices converge to the vertices of the solution with a convergence factor given by the attraction factor $\kappa$.

Proof. Clearly, $C_{d}=1$. Now apply Theorem 2.7 and the estimate (2.10).

Method 3.3. This is the method defined by the operator $K_{l}, \mathrm{cf}$. (2.7). In this method the curve is approximated by the polygon itself. In view of Remark 2.10 the theoretical results for this method are very much like the results for Method 3.1. The error in the vertices of a solution is proportional to the maximum of the squares of the radial distances between $\gamma$ and chords passing through $\gamma\left(\hat{\theta}_{j}\right), \gamma\left(\hat{\theta}_{j+1}\right)$, cf. Remark 2.10. That is, the method is a second-order method. However, the method is not exact if $\gamma$ is a circle.

The Method 3.3 has a simple property too. It is not related to accuracy, but rather to the position of the approximating polygon relative to $\gamma$. The property holds true in $\mathbb{R}^{2}$ for convex curves $\gamma$. The precise formulation is as follows.

LEMMA 3.4. Let $\mathbf{p}\left(\left\{x_{i}\right\}_{i=1}^{N}\right)$ be a polygon approximating the convex invariant Jordan curve $\gamma$. Let the exterior of $\gamma$ be mapped into the exterior, and the interior into the interior. Let every vertex of $\mathbf{p}\left(\left\{x_{i}\right\}_{i=1}^{N}\right)$ belong to a line segment $\left[\Phi x_{j}, \Phi x_{k}\right]$. Then $\mathbf{p}\left(\left\{x_{i}\right\}_{i=1}^{N}\right)$ is in the interior of $\gamma$ or on $\gamma$.

Proof. Assume the contrary, and let $x_{i}$ be in the exterior $\Omega$ of $\gamma$. Then $x_{i}$ belongs to a line segment of images of vertices, $x_{i} \in\left[\Phi x_{j}, \Phi x_{k}\right]$. Observe that images in the exterior must have original (vertices) in the exterior. The vertices are separated in two disjoint sets, those in $\Omega$ and those not in $\Omega$. The map $\Phi$ acts on each of these two sets separately. Then, since $x_{i}$ belongs to the exterior $\Omega$, the convexity of $\gamma$ implies

$$
d\left(x_{i} ; \gamma\right) \leq \max _{x_{i} \in \Omega} d\left(\Phi x_{i} ; \gamma\right)
$$

The same estimate holds true for all vertices in the exterior. Hence,

$$
\max _{x_{i} \in \Omega} d\left(x_{i} ; \gamma\right) \leq \max _{x_{i} \in \Omega}\left(\Phi x_{i} ; \gamma\right)
$$

On the other hand, the attractivity of $\gamma$ and the property $\Phi \Omega \subset \Omega$ imply that the right-hand side is smaller than the left-hand side. This is a contradiction. So the assumption was false. That is, there is no vertex of the polygon in the exterior of $\gamma$.

For Poincaré maps of differential equations the assumption about the behavior of $\Phi$ in relation to the exterior and the interior of $\gamma$ follows from a unique solvability assumption for the ordinary differential equation in a neighborhood of the invariant torus, cf. (1.3).

For the Method 3.3 we are able to show that the approximation error does not necessarily admit an asymptotic expansion, not even for regularly distributed abscissae $\hat{\theta}_{j}$. We make this statement more precise in an example. 
Example 3.5. Let $\Phi$ be given in polar coordinates by

$$
\Phi(r, \theta)=(1+\kappa(r-1), \theta+\alpha)
$$

with $0 \leq \kappa<1$ and $\alpha \in(0,2 \pi]$. Thus the map $\Phi$ has the unit circle as invariant circle. This invariant circle is attractive and the circle map $\left.\Phi\right|_{\gamma}$ is a rigid rotation over the angle $\alpha$. The rotation number of the circle map is $\alpha / 2 \pi$. Apply the Method 3.3 with center $x_{C}=0$. The initial polygon is chosen as a regular polygon with $N$ vertices also centered at the origin. In this simple case the solution of the equation $\mathbf{p}\left(\left\{x_{i}\right\}_{i=1}^{N}\right)=\mathbf{p}\left(\left\{K x_{i}\right\}_{i=1}^{N}\right)$ can be computed explicitly. A special case occurs if $\alpha=2 \pi k / N$ for integer $k$. In this special case the solution is a regular polygon with vertices on the unit circle. So the vertices are exact in this special case.

In the generic case, let $\psi=\alpha \bmod \frac{2 \pi}{N}$. Then the radius $r_{N}$ of a vertex of the solution (the solution is a regular polygon) is easily computed, making use of elementary geometry. The law of sines can be used advantageously. The result is

$$
r_{N}=\frac{(1-\kappa) \cos \frac{\pi}{N}}{\cos \left(\psi-\frac{\pi}{N}\right)-\kappa \cos \frac{\pi}{N}} .
$$

Clearly, $r_{N} \leq 1$, in agreement with Lemma 3.4. Also, $r_{N}=1+O\left(1 / N^{2}\right)$, in agreement with (2.10).

The example also indicates that an asymptotic expansion of the error in powers of $1 / N$ does not exist. To see why, let $\alpha=2 \pi \underset{q}{p}$, with $p, q \in \mathbb{N}$ and $q$ a prime number. Then $\psi^{\prime}=\psi / \pi$ is equal to

$$
\psi^{\prime}=\frac{1}{N}\left(k \frac{p}{q}-\operatorname{entier}\left(k \frac{p}{q}\right)\right), \quad k=N \bmod q .
$$

Thus, $\psi^{\prime}$ equals a periodic function in $N$ divided by $N$. This contradicts an asymptotic expansion of $\psi$ and hence of $r_{N}$ in powers of $1 / N$. Thus, Richardson extrapolation seems not applicable.

Definition 3.6. Let $\hat{\theta}_{j}, j=1, \ldots, N$, be abscissae on $[0,2 \pi)$ with $\hat{\theta}_{j}<\hat{\theta}_{j+1}$ for all $j$. We identify 0 and $2 \pi$, thus transforming the interval to a circle. On this circle we interpolate at a point $\theta$ by means of a piecewise cubic Lagrange interpolation polynomial such that (on the circle)

$$
\hat{\theta}_{j-1}<\hat{\theta}_{j}<\theta<\hat{\theta}_{j+1}<\hat{\theta}_{j+2} \text {. }
$$

This interpolation operator will be called $\Pi_{3}$.

LEMMA 3.7. Assume

$$
\mu=\max _{j, k=j \pm 1} \frac{\hat{\theta}_{j}-\hat{\theta}_{j-1}}{\hat{\theta}_{k}-\hat{\theta}_{k-1}} .
$$

Then

$$
\frac{5}{2} \mu \geq\left|\Pi_{3}\right| \geq 1+\frac{\mu^{2}}{2(\mu+1)} .
$$

Proof. Consider the interpolation polynomial of degree $\leq 3$ on the abscissae $-1 / \mu, 0,1,1+1 / \mu$ with function values $-1,1,1,-1$, respectively. This interpolation polynomial is a parabola with maximum value

$$
1+\frac{\mu^{2}}{2(\mu+1)}
$$


attained at $1 / 2$. By means of the Lagrange form and some rough estimates one obtains the upper bound.

Method 3.8. This method is obtained if we use the piecewise cubic interpolation operator $\Pi_{3}$ introduced above. In principle, the method defined by this interpolation operator is capable of an approximation error of order $O\left(\max _{j}\left|\hat{\theta}_{j}-\hat{\theta}_{j+1}\right|^{4}\right)$. However, there may be some problem with the quantity $|\Pi|_{m}$, cf. (3.3). Without additional information about the distribution of the $\hat{\theta}_{j}$, not much can be said.

As a fourth example we consider interpolation by the classical cubic spline. In this instance, a closed curve, we have a periodic spline. First we give an estimate related to the norm of this interpolation operator $\Pi_{s}$.

LEMMA 3.9. Let

$$
\nu=\max _{i, j} \frac{\hat{\theta}_{j+1}-\hat{\theta}_{j}}{\hat{\theta}_{i+1}-\hat{\theta}_{i}} .
$$

Then the norm of the spline interpolation operator is bounded by

$$
\left|\Pi_{s}\right| \leq 1+3 \nu^{2}
$$

Proof. For a simple account of cubic spline interpolation we refer to Stoer and Bulirsch [12, Chapter II]. We give a scalar analysis, but the results carry over to more dimensions. Let the function values in the abscissae $\hat{\theta}_{j}$ be denoted by $f_{j}$. Step sizes are denoted by $h_{j}=\hat{\theta}_{j}-\hat{\theta}_{j-1}$, taking into account the identification of indices 1 and $N+1$. Then, by the formulas given in Stoer and Bulirsch [12] we find for the moments $M_{j}$ the upper bound

$$
\left|M_{j}\right| \leq 12 \frac{\max _{j}\left|f_{j}\right|}{\min _{j} h_{j}^{2}} .
$$

With these estimates for the moments we may estimate the spline itself. Between two successive abscissae the second derivative is a linear function. The moments $M_{j}$ represent the second derivative of the spline in the abscissae. Hence, the second derivative on a subinterval $\left[\hat{\theta}_{j}, \hat{\theta}_{j+1}\right]$ is bounded in modulus by the upper bound for the moments. On the subinterval, write the spline as the sum of a linear function (piecewise linear interpolation) and a cubic part which vanishes at the endpoints $\hat{\theta}_{j}$ and $\hat{\theta}_{j+1}$. This cubic part is the solution of a boundary value problem, and as such it is easily estimated. The linear part is estimated by the maximum of the modulus of the function values. The sum of these two estimates gives the upper bound

$$
\max _{j}\left|f_{j}\right|+\frac{1}{4} \max _{j} h_{j}^{2} \max _{j}\left|M_{j}\right| \text {. }
$$

This results in the estimate (3.5).

Observe that the estimate (3.5) involves the global quantity $\nu$, whereas the estimate for the norm of the piecewise cubic interpolation operator uses the locally defined quantity $\mu$. Clearly, $\nu \geq \mu$. In some instances a lower bound for $\left|\Pi_{s}\right|$ may be obtained rather easily. We give an example.

Example 3.10. Consider an equidistant mesh with $4 M$ abscissae. On this mesh we consider a periodic spline as in Figure 3.1. On each of the subintervals this spline is given either by $\pm \phi_{i}$ or by $\pm \psi_{i}$. The polynomials $\phi_{i}$ and $\psi_{i}$ are defined as 
follows. Let $m_{i}$ be the midpoint of the $i$ th subinterval, and let $\xi=\left(x-m_{i}\right) / h, h$ the mesh spacing. Then, on the subinterval of which $m_{i}$ is the midpoint,

$$
\phi_{i}(\xi)=\frac{11}{8}-\frac{3}{2} \xi^{2}, \quad \psi_{i}(\xi)=\frac{9}{4} \xi-\xi^{3} .
$$

When put in the right order with the right sign, these functions define a spline. The values at the abscissae are \pm 1 , the values at the midpoints are 0 and $\pm 11 / 8$. Hence $\left|\Pi_{s}\right| \geq 11 / 8$ on equidistant abscissae.

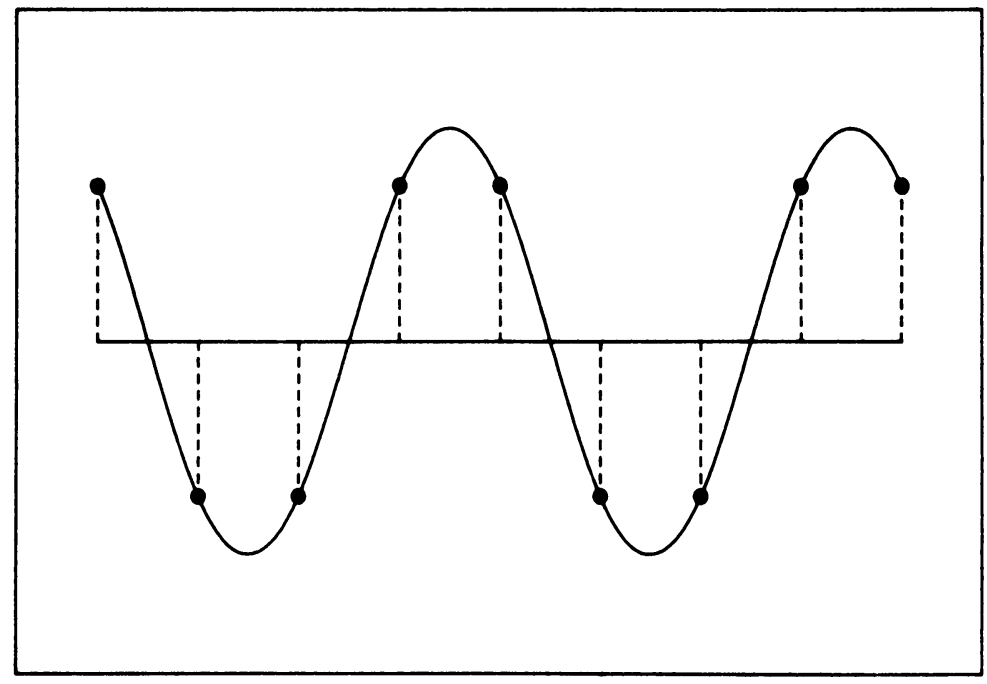

FIGURE 3.1

Part of the periodic cubic spline of Example 3.10.

Method 3.11. This is the method obtained by choosing cubic spline interpolation. In view of Theorem 2.9 this method is of fourth order. The large norm of the interpolation operator may cause some problems, as is the case in Method 3.8.

The theory of Section 2 explains why these methods give good results for easy problems, with nearly equidistant $\hat{\theta}_{j}$. The methods are perhaps not as robust as Method 3.1 or Method 3.3. This may also be true for other higher-order methods, e.g., piecewise interpolation by fifth-degree polynomials, B-spline interpolation of higher degree, see Schumaker [11]. These interpolation operators are defined by means of divided differences, and the divided differences might induce large interpolation operator norms for nonuniform mesh spacings. Observe that we use a projection property in the definition of $K$. So we need the B-spline approximation obtained via a projection, cf. Schumaker [11].

Let us now apply the methods to simple examples. The first example is given by the map (3.1). So the unit circle is an invariant circle. For this map tubular coordinates and polar coordinates differ only in a shift over a distance 1 in the radial component. We shall compute the constant $C_{d}$ which relates the tubular distance to $\gamma$ to the radial distance to $\gamma$. We do this for infinitesimal deviations from the

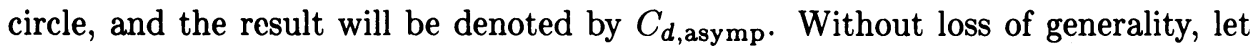

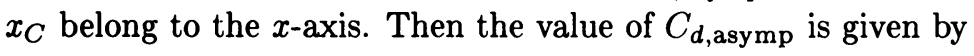

$$
C_{d, \text { asymp }}=\max _{\tau} \frac{1}{\cos (\sigma)},
$$


where the angle $\sigma$ is determined by $x_{C}$ and $\tau$ as in Figure 3.2. It turns out that this maximum can be computed explicitly as a function of $x_{C}$. Again, the law of sines is useful. After some computations the result is

$$
C_{d, \text { asymp }}=\left(1-x_{C}^{2}\right)^{-1 / 2} \text {. }
$$

This result says that $C_{d}$ is not too large, if $x_{C}$ is not close to $\gamma$.

We are also able to compute the factor $\bar{\kappa}_{\text {asymp }}$, i.e., the limiting value of the factor $\bar{\kappa}$, cf. Assumption 2.8, when the width of the annular neighborhood tends to zero. The value of $\bar{\kappa}_{\text {asymp }}$ depends only on $x_{C}$ and $\kappa$. It is easily seen that $\bar{\kappa}_{\text {asymp }}$ is given by (cf. Figure 3.2 where the situation $\alpha=0$ has been sketched)

$$
\bar{\kappa}_{\text {asymp }}=\max _{\tau} \lim _{y \rightarrow x} \frac{\|\Phi y-x\|}{\|x-y\|} .
$$

This limit can be computed by goniometric formulas. We find

$$
\bar{\kappa}_{\text {asymp }}=\max _{\tau}\left\{\kappa^{2} \cos ^{2}(\sigma)+\sin ^{2}(\sigma)\right\}^{1 / 2} .
$$

This results in the estimate, cf. (3.6), (3.7),

$$
\bar{\kappa}_{\text {asymp }}=\left\{\kappa^{2}\left(1-x_{C}^{2}\right)+x_{C}^{2}\right\}^{1 / 2} .
$$

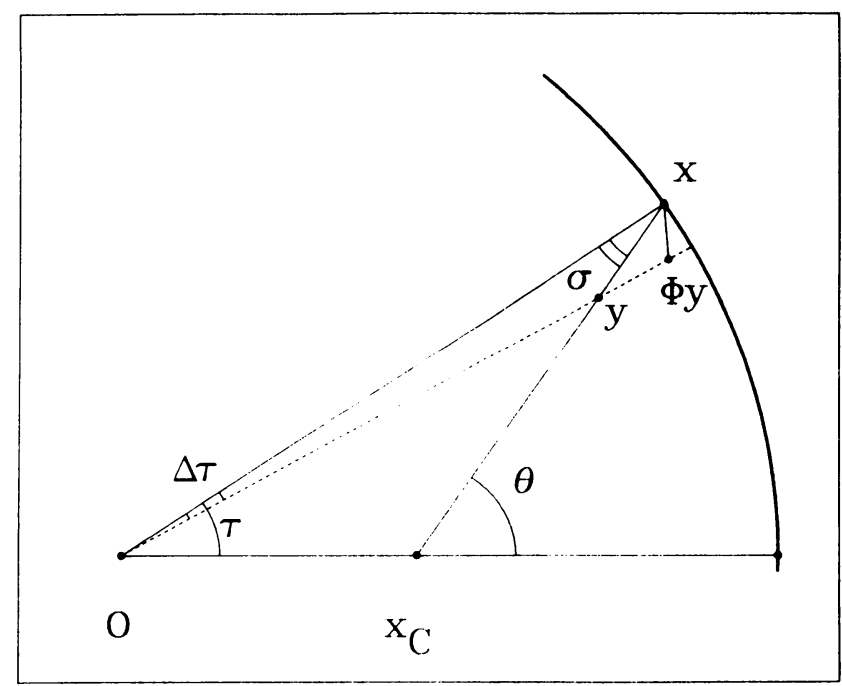

FiguRE 3.2

The correspondence between the $\tau$-component of the tubular coordinates, the $\theta$-component of the radial coordinates and the angle $\sigma$ for points on the circle $\gamma$.

This implies $\bar{\kappa}_{\text {asymp }}<1$ for $\kappa<1$ and $0 \leq x_{C}<1$, which is a rather satisfactory result. It says that Assumption 2.8 is valid in this simple example, provided the neighborhood of $\gamma$ is sufficiently narrow. For annular neighborhoods of $\gamma$ with finite width one may compute $\bar{\kappa}$ numerically. Here we only mention the result of such tests: the asymptotic formula for $\bar{\kappa}$ is rather accurate for $x_{C}$ not too close to $\gamma$.

In the numerical example we choose $\kappa=4 / 5$ and $\alpha=0.1$. In addition, we choose (almost) equidistant initial estimates on the circle. However, if $x_{C}$ is not in the center of the circle, then the abscissae $\hat{\theta}_{j}$ are not equidistant. Observe that the 
quantities $\mu$, cf. (3.2), and $\nu$, cf. (3.4), increase if $x_{C}$ moves away from the center of the circle. So do the (upper bounds of the) norms of the interpolation operators used in Method 3.8 and Method 3.11, respectively. For Method 3.1 and Method 3.3

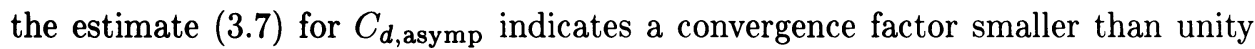
for $\left|x_{C}\right|<0.6$. For Method 3.8 and Method 3.11 we do not obtain a (predicted) convergence factor smaller than unity.

The estimates for $\bar{\kappa}|\Pi|_{m}$, cf. Theorem 2.9, based on the asymptotic formula (3.8) give different results. For Method 3.1 and Method 3.3 we obtain a convergence factor smaller than unity for all $x_{C} \in[0,1)$. For Method 3.8 and Method 3.11 the estimated convergence factors are not smaller than unity. For these methods (and $\kappa=4 / 5$ ) the norm of the interpolation operator spoils the convergence factor $\bar{\kappa}_{\text {asymp }}<1$.

In the iterative process we compute the norm $\Delta_{i}$ of the difference of two successive iterates (= vertices of polygons). The process stops as soon as $\Delta_{i}<1.0 \mathrm{E}-08$ and $\Delta_{i+1}<1.0 \mathrm{E}-08$. The convergence factor $\varrho$ is determined from all iterates with the property $\Delta_{i}<1.0 \mathrm{E}-06$. In a computer arithmetic of approximately 14 decimal digits there are no problems with rounding errors. The initial vertices are on a regular polygon with radius 1 with an offset of $5.0 \mathrm{E}-04$ from the origin. Results have been computed for discrete values of $x_{C}$ at the points $i / 100, i=0,1 \ldots$ For Method 3.11 convergence has been obtained for $0 \leq x_{C}<0.91$, for Method 3.8 the range was $0 \leq x_{C} \leq 0.84$, for Method 3.1 the range was $0 \leq x_{C} \leq 0.99$, and for Method 3.3 the range was $0 \leq x_{C} \leq 0.97$. The numerical estimates for $\varrho$ are sketched in Figure 3.3. The results for Method 3.1 and Method 3.3 are almost identical; that is why the results for Method 3.3 are not shown. This experiment for a very simple problem indicates the different behavior of Method 3.8 and Method 3.11 in contrast to the two other methods. Method 3.8 does not converge as rapidly as the low-order ones, at least not for $x_{C}>3 / 5$. Method 3.11 does rather well as compared with Method 3.8, at least in this simple example. Also, the results indicate that the estimates of Section 2 are too pessimistic.

\section{TABLE 1}

Results for the map (3.1) with $\kappa=\alpha=0.1$. The table gives the error in the approximation for various values of $N$ and the order of accuracy.

\begin{tabular}{r|c|c|c|c}
\hline \multicolumn{1}{c|}{$N$} & Method 3.1 & Method 3.3 & Method 3.8 & Method 3.11 \\
\hline 20 & $1.190 \mathrm{E}-02$ & $2.387 \mathrm{E}-03$ & $2.413 \mathrm{E}-04$ & $1.162 \mathrm{E}-05$ \\
40 & $3.163 \mathrm{E}-03$ & $9.391 \mathrm{E}-04$ & $2.492 \mathrm{E}-05$ & $2.079 \mathrm{E}-06$ \\
80 & $6.809 \mathrm{E}-04$ & $2.900 \mathrm{E}-04$ & $2.118 \mathrm{E}-06$ & $2.057 \mathrm{E}-07$ \\
160 & $2.116 \mathrm{E}-04$ & $9.719 \mathrm{E}-05$ & $1.896 \mathrm{E}-07$ & $2.078 \mathrm{E}-08$ \\
320 & $1.894 \mathrm{E}-05$ & $9.449 \mathrm{E}-06$ & $4.367 \mathrm{E}-09$ & $1.967 \mathrm{E}-10$ \\
\hline$p$ & 2.25 & 1.92 & 3.85 & 3.83 \\
\hline
\end{tabular}


Method 3.1, Method 3.8 and Method 3.11 do not give the exact solution for this problem unless $x_{C}=0$. For $x_{C}=\frac{1}{2} \sqrt{2}, \kappa=0.1, \alpha=0.1$ we compute the error in the vertices as the maximal distance to the unit circle. The results are given in Table 1 . The order of accuracy $p$ may be estimated by plotting the results on a double logarithmic scale. The order is given in the bottom line of Table 1. These order results are more or less in agreement with the theoretical prediction. The rather good agreement is somewhat surprising, since we have no asymptotic expansion for the error (at least not for Method 3.3).

As a second example we consider the delayed logistic map

$$
\Phi(\xi, \eta)=(\eta, \lambda \eta(1-\xi)) .
$$

This map has been investigated in great detail by Aronson et al. [1]. For $\lambda>2$ there is an attracting invariant curve, at least until $\lambda \sim 2.20$. For $\lambda=2.11$ we apply the four methods of this section to this problem. The invariant curve is convex and smooth for this parameter value. The source in the interior of the curve is the point $(\sigma, \sigma)$, where $\sigma=(\lambda-1) / \lambda$. We choose $x_{C}=(\sigma, \sigma)$. For all four methods we use the same initial guess. This initial guess is in fact a good approximate to the curve, with nicely distributed vertices. For this good initial guess the four methods converge. We use $\Phi^{11}$ instead of $\Phi$ itself. By inserting new vertices in the middle of line segments we are able to double the number of vertices without changing the shape of the mesh, i.e., without changing the distribution of the angles $\theta_{j}$ over the circle. In this way we double the mesh in Table 2.

\section{TABLE 2}

Results for the delayed logistic map (3.9) with $\lambda=2.11$. The table gives the error for various values of $N$. The error is measured as explained in the text. The last line gives the observed order of accuracy.

\begin{tabular}{r|c|c|c|c}
\hline \multicolumn{1}{c|}{$N$} & Method 3.1 & Method 3.3 & Method 3.8 & Method 3.11 \\
\hline 36 & $4.385 \mathrm{E}-04$ & $1.725 \mathrm{E}-03$ & $1.023 \mathrm{E}-04$ & $1.250 \mathrm{E}-05$ \\
72 & $1.325 \mathrm{E}-04$ & $3.359 \mathrm{E}-04$ & $6.450 \mathrm{E}-06$ & $1.395 \mathrm{E}-07$ \\
144 & $3.004 \mathrm{E}-05$ & $9.415 \mathrm{E}-05$ & $3.209 \mathrm{E}-07$ & $2.826 \mathrm{E}-08$ \\
288 & $8.480 \mathrm{E}-06$ & $2.137 \mathrm{E}-05$ & $1.713 \mathrm{E}-08$ & $2.363 \mathrm{E}-09$ \\
\hline$p$ & 1.92 & 2.08 & 4.20 & 3.94 \\
\hline
\end{tabular}

We compute points on the curve $\gamma$ as follows. We need these points for estimating the approximation error. First, from the convergence of Method 3.3 for the invariant curve of $\Phi^{11}$, we find a convergence rate of approximately 0.5 . This means that the attraction factor $\kappa$ is approximately $0.5^{1 / 11} \sim 0.94$. Hence, for a point within a distance of 0.1 from the curve, the 900 th iterate is within a distance of $6.5 \mathrm{E}-26$ from the curve. That is, in exact arithmetic. In the presence of rounding errors we 
expect that the distance to the curve is of the order of the machine precision. The error in the position on the curve may build up, but that is of no interest in the present discussion. Hence, if we start with a point $x_{0}$ almost on the curve, then the points $x_{j}=\Phi^{j} x_{0}, j=971, \ldots, 1000$ form a set of thirty points on the curve, within working precision. We now estimate the error in the results by computing the maximal radial distance from these thirty points to the approximating curve. This is the error mentioned in Table 2. The order of accuracy agrees quite well with the theoretical order of accuracy.

Finding an initial guess is somewhat more difficult for Method 3.8 and Method 3.11 than for the simpler ones. However, once a good initial guess is obtained, pathfollowing methods combine quite well with each of the four methods. For example, increasing $\lambda$ by 0.02 at a time, a solution for $\lambda=2.19$ is easily obtained for Method 3.11. See Doedel and Kernevez [4] and Rheinboldt [10] for efficient path-following techniques. A similar observation can be made for a nonlinear recurrence relation mentioned in Koçak [8] (the difference equation quad 2). Without a good initial guess, a method like Method 3.11 does not converge. But with a good guess, the method converges and the results are rather good, much better than the results for the second-order methods.

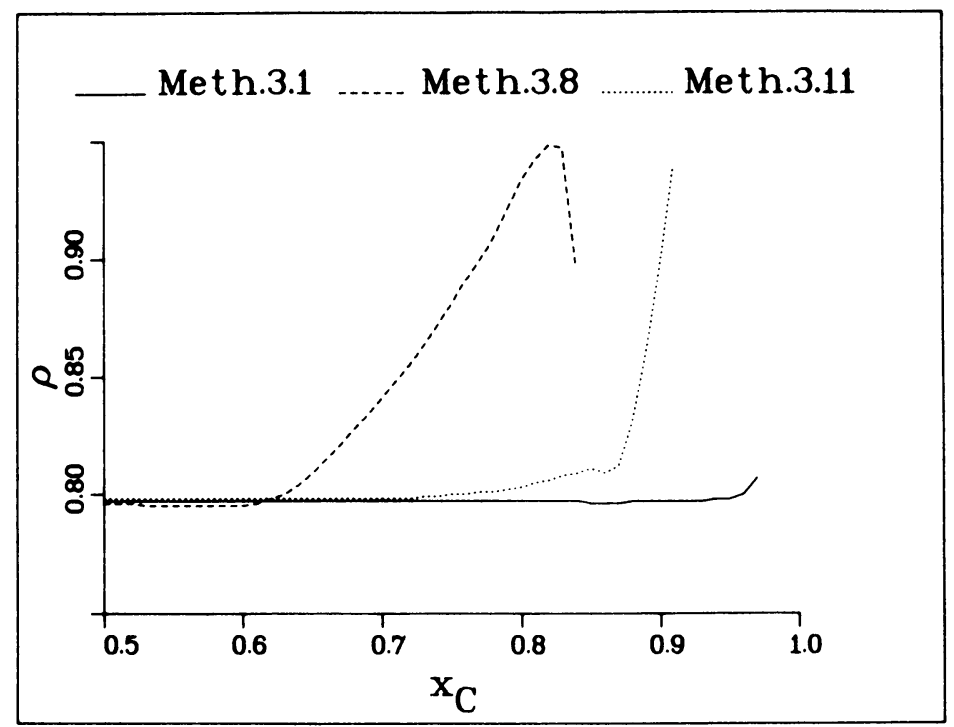

FIgURE 3.3

Results obtained for the map (3.1) with $\kappa=0.8, \alpha=0.1$. The value of $x_{C}$ is shown on the horizontal axis, the rate of convergence $\varrho$ is shown on the vertical axis. For $0 \leq x_{C}<\frac{1}{2}$ the observed rate of convergence is $\mathbf{0 . 8}$ for all three methods. The results for Method 3.3 are almost identical to the ones shown for Method 3.1. 


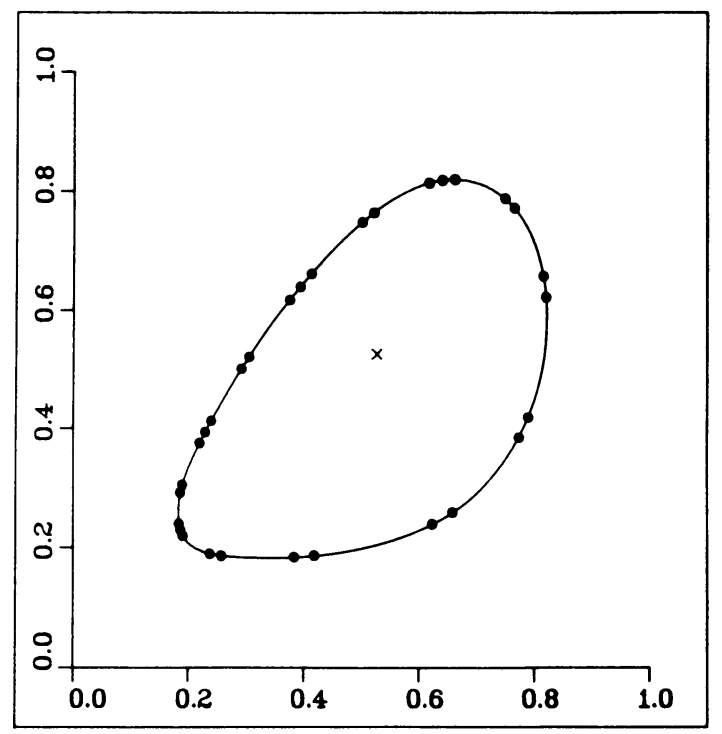

FIGURE 3.4

Invariant curve for the delayed logistic map (3.9) for $\lambda=2.11$. The $\xi$-axis is the horizontal one. The black dots on the curve are the points in which the error in the radial direction of the methods is measured. The point in the interior is the source $\left(=x_{C}\right)$.

Department of Mathematics and Computer Science

Vrije Universiteit

De Boelelaan 1081

1081 HV Amsterdam, The Netherlands

1. D. G. ARonson, M. A. Chory, G. R. HALl, \& R. P. MCGeEhee, "Bifurcations from an invariant circle for two-parameter families of maps of the plane," Comm. Math. Phys., v. 83, 1982, pp. 303-354.

2. R. Bouc, M. DEFilippi \& G. IOOss, "On a problem of forced nonlinear oscillations. Numerical example of bifurcation into an invariant torus," Nonlinear Anal., v. 2, 1978, pp. 211224.

3. Tze Ngon Chan, Numerical Bifurcation Analysis of Simple Dynamical Systems, Thesis (unpublished), Dept. of Computer Science, Concordia University, Montreal.

4. E. J. Doedel \& J. P. KeRnevez, "Software for continuation problems in ordinary differential equations with applications," Preprint.

5. J. Guckenheimer \& Ph. Holmes, Nonlinear Oscillations, Dynamical Systems and Bifurcations of Vector Fields, Springer-Verlag, New York, 1983.

6. J. K. HALE, Ordinary Differential Equations, Wiley-Interscience, New York, 1969.

7. I. G. Kevrekidis, R. ARIS, L. D. Schmidt, \& S. PEliKan, "Numerical computation of invariant circles of maps," Phys. D, v. 16, 1985, pp. 243-251.

8. H. KOÇAK Differential and Difference Equations through Computer Experiments, SpringerVerlag, New York, 1986.

9. N. LEVINSON, "Small periodic perturbations of an autonomous system with a stable orbit," Ann. of Math., v. 52, 1950, pp. 727-738.

10. W. C. Rheinboldt, Numerical Analysis of Parametrized Nonlinear Equations, Wiley, New York, 1986.

11. L. SChUMAKER, Spline Functions: Basic Theory, Wiley, New York, 1981.

12. J. StOER \& R. BUliRSCH, Introduction to Numerical Analysis, Springer-Verlag, Berlin and New York, 1980.

13. E. ThOULOUZE-PRATT, "Existence theorem of an invariant torus of solutions to a periodic differential system," Nonlinear Anal., v. 5, 1981, pp. 195-202. 
14. E. Thoulouze-Pratt \& M. Jean, "Analyse numérique du comportement d'une solution presque périodique," Internat. J. Non-Linear Mech., v. 17, 1982, pp. 319-326.

15. M. VAN VELDHUIZEN, "A new algorithm for the numerical approximation of an invariant curve", SIAM J. Sci. Statist. Comput., v. 8, 1987, pp. 951-962.

16. M. VAN VELDHUizen, "On the numerical approximation of the rotation number," J. Comput. Appl. Math., v. 21, 1988, pp. 203-212. 